\title{
Socioeconomic factors, psychological factors, and function in adults with chronic musculoskeletal pain from rural Nepal
}

This article was published in the following Dove Press journal: Journal of Pain Research

\author{
Saurab Sharma ${ }^{1,2}$ \\ Anupa Pathak ${ }^{1,3}$ \\ Jyoti Jha ${ }^{1,4}$ \\ Mark $P$ Jensen ${ }^{5}$ \\ 'Department of Physiotherapy, \\ Kathmandu University School \\ of Medical Sciences, Dhulikhel, \\ Nepal; ${ }^{2}$ Center for Musculoskeletal \\ Outcomes Research, Department of \\ Surgical Sciences, Dunedin School \\ of Medicine, University of Otago, \\ Dunedin, New Zealand; ${ }^{3}$ School of \\ Physiotherapy, University of Otago, \\ Dunedin, New Zealand; ${ }^{4}$ Department \\ of Physiotherapy, Grande International \\ Hospital, Kathmandu, Nepal; \\ ${ }^{5}$ Department of Rehabilitation \\ Medicine, University of Washington, \\ Seattle, WA, USA
}

Background: Both socioeconomic and psychological factors have been shown to predict patient function in samples of individuals with chronic pain in Western countries. However, little is known about their role as predictors of function in individuals with chronic pain from developing countries.

Purpose: The purpose of this study was to examine the associations between measures of socioeconomic factors (income, education) and psychological factors (catastrophizing and resilience) and measures of function in a sample of individuals with chronic pain from rural Nepal. In addition, we sought to evaluate the moderating effects of socioeconomic factors on the associations between the psychological variables and function.

Methods: We interviewed 143 adults with chronic musculoskeletal pain from rural areas of Nepal to assess income, education level, pain intensity, catastrophizing, resilience, physical function, and depression. We performed two regression analyses to evaluate the direct and unique effects of the socioeconomic and psychological variables and pain intensity as predictors of patient function, as well as the moderating influence of income, education level, and pain intensity on the associations between the psychological variables and function.

Results: Education and income both predicted physical function, but only income predicted depression. In addition, pain catastrophizing, but not resilience, evidenced a direct and significant independent association with depression. Neither catastrophizing nor resilience made independent and significant direct contributions to the prediction of physical function. The association between resilience and physical function was moderated by pain intensity and income, and income (but not education or pain intensity) moderated the associations between both 1) resilience and depression and 2) catastrophizing and depression.

Conclusion: The results suggest the possibility that cultural differences may influence the role that psychosocial factors play in chronic pain adjustment. These findings have important implications regarding how psychosocial pain interventions should be adapted by individuals in developing countries.

Keywords: chronic pain, musculoskeletal pain, culture, depression, resilience, pain catastrophizing

\section{Introduction}

Biopsychosocial models of chronic pain hypothesize important roles of psychological factors as contributors to pain and its impact on individuals with chronic pain. ${ }^{1-5}$ Two psychological factors that have been the focus of previous research in this area are pain catastrophizing and resilience. Pain catastrophizing can be defined as ruminating about, and focusing on, overly negative beliefs about pain and its impact. ${ }^{6}$ Pain catastrophizing 
tends to show a fairly consistent positive association with measures of psychological dysfunction in individuals with chronic pain $;^{7-9}$ its association with disability, while sometimes strong, is less consistent. ${ }^{10-12}$ Resilience can be defined as an individual's ability to recover or "bounce back" from negative events or maintain function (or even thrive and grow) in the face of ongoing stress. ${ }^{13}$ Although research on the role that resilience plays in adjustment to pain is relatively new, the findings that are available provide preliminary support for the importance of resilience to function in individuals with chronic pain. ${ }^{13-18}$

In addition, and especially given the variability in the strength of the association between catastrophizing and disability found in previous research, the impact of catastrophizing and resilience on chronic pain adjustment could potentially be influenced by a number of contextual factors. For example, people living with chronic pain in rural areas of the US have been shown to report higher levels of pain intensity, endorse more maladaptive beliefs about pain, use more so-called maladaptive pain-coping strategies, and endorse higher levels of pain catastrophizing than individuals from urban areas. ${ }^{19}$ To the extent that these differences in mean values influence or are associated with differences in the variability of catastrophizing or resilience in individuals living in rural vs urban areas, where people live could potentially moderate the strength of the associations between these psychosocial factors and adjustment to chronic pain. ${ }^{20}$

Socioeconomic status (SES) is another factor that could potentially moderate the association between psychological factors, such as pain catastrophizing and resilience, and measures of physical and psychological functions in individuals with chronic pain. For example, individuals with a lower SES have fewer resources available to them and so may need to continue to work despite having pain or regardless of their levels of pain catastrophizing or resilience, limiting the impact of these psychosocial variables on function. If this were true, then pain catastrophizing and resilience would be expected to have a weaker association with function among individuals with very low levels of SES, relative to individuals with a higher SES. In partial support of this idea, one study conducted in the US found that the association between catastrophizing and psychological distress - but not catastrophizing and physical function - was moderated by both social function and education level in a sample of individuals with rheumatoid arthritis. ${ }^{21}$ However, the potential moderating effects of income level were not evaluated in this study. Another recent study conducted in Spain found that pain intensity moderated the relationship between pain catastrophizing and physical function but did not play a role in the relationship between pain catastrophizing and psychological function in a sample of patients with chronic pain. ${ }^{22}$ To our knowledge, no studies have yet examined the potential moderating effects of pain intensity and SES on the association between resilience and function in either developed or developing countries and moderating role of SES in the relation of catastrophizing and function in developing nations.

Culture may be another contextual factor that could impact the role that pain catastrophizing or resilience may play in adjustment to chronic pain. Evidence supporting this possibility comes from research showing important differences in associations between key psychosocial variables - such as pain-related beliefs and coping - and measures of adjustment in individuals with chronic pain who are not from the USA, relative to individuals with chronic pain from the USA. ${ }^{23,24}$

The vast majority of research studies examining the roles of socioeconomic and psychosocial factors in chronic pain adjustment come from research using participants from developed North American and European ${ }^{10,12,14,25}$ and Asian countries. ${ }^{26-31}$ Research on the role that these and other psychosocial factors play in adjustment to chronic pain in developing countries is relatively scarce. In two studies evaluating the psychometric properties of measures of catastrophizing and resilience in individuals with chronic pain from Nepal (who were also participants in the current analyses), we found significant and moderate univariate associations between measures of both domains and depression. ${ }^{28,29}$ However, to our knowledge, no studies have examined the unique associations between resilience and physical function when controlling for catastrophizing (or between catastrophizing and physical function with controlling for resilience) in the same study in any country, and no studies have examined the role that socioeconomic variables play in adjustment to chronic pain in developing countries.

Research examining the role that socioeconomic and psychological factors play as predictors of patient function in individuals with chronic pain from Nepal would contribute to our understanding of the extent to which findings in patients from developed countries replicate (or do not replicate) in patients from developing countries. Importantly, people in developing countries - in particular, Nepalese individuals who live in rural areas - differ from people in developed countries in a number of important ways. First, they have an extremely low SES (average annual income is $\$ 718$, expressed in US dollars, or $€ 648$, expressed in euros) $)^{32}$ and tend to have very low education levels (only 10\% of the Nepalese population have completed 10 years or more of education). ${ }^{33}$ People from 
Nepal also differ from those from Western countries in terms of their culture and religion, as the predominant religion in Nepal is Hinduism; ${ }^{33}$ to our knowledge, no previous research examining pain catastrophizing or resilience in individuals with chronic pain has been performed in samples of individuals who were predominantly Hindu.

The current study sought to address the knowledge gaps in this area by 1) examining the associations between socioeconomic variables (income and education) and pain intensity, psychological variables (pain catastrophizing and resilience), and measures of physical and psychological function (depression) in a sample of individuals with chronic pain from rural areas of Nepal and 2) understanding the role that socioeconomic variables and pain intensity may play in moderating the associations between psychological variables and function in the study sample. Based on the idea that findings from research performed in developed countries would replicate in a study using data from individuals from Nepal, we hypothesized that individuals with chronic pain from Nepal who endorsed more pain catastrophizing and less resilience would also report higher levels of depression and physical disability, relative to individuals who endorsed lower levels of pain catastrophizing and more resilience. We also anticipated that education level and income would have significant independent associations with function, with both evidencing significant and positive associations with physical function but negative associations with depression. Finally, we did not have a priori hypotheses regarding these potential moderation effects, given the lack of any previous research that has examined these effects.

\section{Methods}

\section{Design and setting}

This was a secondary analysis of a cross-sectional study dataset used to report the validity of the Nepali versions of measures of resilience and catastrophizing. ${ }^{34,35}$ We recruited participants from two sites: 1) patients from rural areas seeking care in a tertiary care hospital and 2) individuals with chronic pain residing in a rural community of Nepal. The tertiary care hospital from which the hospital subset of the study sample was recruited is located 30 kilometers from Kathmandu in the Kavre district and primarily serves Kavre and other rural districts. We conducted a door-to-door survey, identified individuals with chronic pain, and interviewed them in the rural community sample subset in the Dolakha district of Nepal. Ethical approval was obtained from the institutional review committee of Kathmandu University School of Medical Sciences (reference number 75/15).

\section{Participants}

Data provided by the participants from this study have also been used to evaluate the psychometric properties of the measures of catastrophizing and resilience used in this study. ${ }^{34,35}$ Although both of the previous studies reported on the univariate associations between catastrophizing and resilience and depression, neither reported on the associations between these predictors and physical function, and neither evaluated the moderating effects of income and education on these associations, which are aims of the current study.

In order to be eligible to participate, potential participants needed to 1) have self-reported or physician-diagnosed chronic musculoskeletal pain (identified by using body chart) with a complaint of pain for more than half of the days in the past 3 months; 2) be a citizen of Nepal and able to speak Nepali fluently; 3) be 18 years or older; 4) have pain for a minimum of 3 months; and 5) report an average pain intensity in the past week of $\geq 4$ on a $0-10$ Numerical Pain Rating Scale, where 0 is "no pain" and 10 is "maximum pain.” Exclusion criteria were: 1) having an acute medical problem that caused the pain, such as a diagnosis of an infection or cancer; 2) having a recent trauma that caused the pain (eg, fracture); and 3) an inability to communicate in Nepali. After screening the potential participants for eligibility, research staff explained the study procedures to eligible participants. Written informed consent was obtained from all the participants before the start of the study if the participants could read and write. Verbal consent was obtained from those participants who could not read or write, and a witness signed on their behalf. All the study measures (described earlier) were then administered via in-person interviews by one of the study authors (SS, AP, or JJ) or by a trained research assistant.

\section{Measures}

\section{Demographic variables}

Participants were asked to provide demographic information; specifically, their age, sex, religion, ethnicity, education, occupation, and income. Their monthly income (in Nepali rupees) was classified as 0 ("No income"), 1 ("Less than or equal to 10,000 rupees"; 10,000 Nepalese rupees is equal to about 93 US dollars or 86 Euros), 2 ("10,001-30,000 rupees”), 3 (“30,001-50,000 rupees”), 4 (“50,001-100000 rupees"), and 5 ("More than 100,000 rupees").

\section{Site(s) of pain}

Participants were asked to indicate the site(s) of their pain using a pain drawing. ${ }^{36}$ Based on their responses to the draw- 
ing, we classified participants as having or not having pain in each of nine body locations: neck, shoulder, elbow, wrist and hand, upper back, lower back and pelvis, knee and foot, ankle, or multiple (more than one) site. The sites of pain were only used to describe the participants in the current study. Pain drawings have demonstrated good reproducibility across a number of samples of individuals with chronic pain. ${ }^{36-39}$

\section{Pain intensity}

Average pain intensity in the last 1 week were assessed using a Nepali version of the 11-point Numerical Rating Scale (NRS) with the anchors 0 ("No pain") and 10 ("Maximum pain"). ${ }^{40}$ Eleven-point NRS scales have demonstrated validity and reliability as measures of pain intensity measure for a larger variety of painful conditions and different age groups and are recommended by consensus groups for use in pain research. ${ }^{37,41,42}$

\section{Pain catastrophizing}

Pain catastrophizing was assessed using a Nepalese version of the 13-item Pain Catastrophizing Scale (PCS) with which participants were asked to rate the frequency with which they think 13 different catastrophizing thoughts using a 5-point Likert scale ranging from 0 ("Not at all") to 4 ("All the time"). 6,35 Scores can range from 0 to 52 with higher scores indicating higher catastrophizing. A great deal of support exists for the reliability and validity of the PCS in individuals with chronic pain..$^{35,43,44}$ The PCS had excellent internal consistency in the current sample (Cronbach's alpha=0.91).

\section{Resilience}

The Nepali version of the two-item version of Connor Davidson Resilience Scale (CD-RISC) was used to assess the resilience. ${ }^{34,45}$ The two items included in this version were "I am able to adapt when changes occur" and "I tend to bounce back after illness, injury or other hardships". The two items were scored on a Likert scale ranging from 0 ("Not true at all") to 4 ("True nearly all the time"). The total scores can range from 0 to 8 , with higher scores indicating more resilience. The measure had marginal internal consistency (Cronbach's alpha $=0.60$ ) in the current sample. The Nepali version of the two-item CD-RISC has evidenced good test-retest stability (intraclass correlation coefficient of 0.70 ), concurrent validity with strong association with the 10 -item longer version scale, and construct validity with weak to moderate negative univariate associations with measures of pain catastrophizing, anxiety, depression, and pain intensity. ${ }^{34}$

\section{Depression}

The Nepali version of the 21-item Beck Depression Inventory $(\mathrm{BDI})^{46,47}$ was used to assess the depression. Respondents were asked to indicate the severity of depression symptom using 4-point scales ranging from 0 to 3. Each 4-point scale response is tailored to each item. Total scores can range from 0 to 63 with higher scores indicating more severe depressive symptoms. A great deal of evidence supports the reliability and validity of the BDI as a measure of depression across a large variety of samples. ${ }^{47-50}$ Studies by Kohrt et al ${ }^{47}$ reported excellent psychometric properties of Nepali version of BDI, with an excellent internal consistency (Cronbach's alpha $=0.90),{ }^{47}$ a high 2 -week test-retest reliability $\left(r=0.84^{51}\right)$, and excellent ability to classify individuals as depressed or not (area under the curve $=0.92,95 \%$ CI $0.88-0.96$ ). The BDI evidenced excellent internal consistency (Cronbach's alpha $=0.90$ ) in the current sample.

\section{Physical function}

To assess physical function, we used the Nepali version of the Patient-Specific Functional Scale (PSFS), ${ }^{52}$ which asks respondents to list three activities that they are unable to do or are having difficulty doing because of chronic pain. Each respondent-generated item is then rated on an 11-point difficulty scale from 0 ("Unable to perform the activity") to 10 (" $100 \%$ able to perform the activity at the same level before your chronic pain state"), ${ }^{53}$ with a total score ranging between 0 and 30 for three items. Higher scores indicate higher levels of physical function. A systematic review of PSFS psychometric literature concluded that the PSFS is a valid and reliable measure for assessing physical function in individuals with chronic musculoskeletal pain conditions. ${ }^{54}$ The testretest reliability of PSFS in the review ranged between 0.76 and 0.97 over a period of 1 day- 5 weeks, indicating adequate to excellent stability in individuals with a chronic low back pain. The construct validity of the PSFS has been supported with moderate to strong associations with measures that have "fixed-items" such as the Roland Morris Disability Questionnaire, Oswestry Disability Index, Lower Extremity Functional Scale, and Upper Extremity Functional Index in individuals with musculoskeletal pain $(r \mathrm{~s}=0.36-0.83) .^{54,55}$ Similarly, the PSFS has been found to be valid for comparison of group-level change and between-group discrimination. ${ }^{55}$ The Nepali version of the PSFS has shown to be both reliable and valid with an internal consistency (Cronbach's alpha) of 0.75 , a test-retest stability (intraclass correlation coefficient) of 0.89 , and construct validity with its associations with the measures of disability and pain intensity. ${ }^{52}$ The internal 
consistency (Cronbach's alpha) in the current sample was 0.88 , indicating good reliability.

\section{Data analysis}

We first computed descriptive statistics of demographic, pain, and pain history variables to describe the sample. Next, we examined the distributions of the study variables (for skewness and kurtosis) and computed the variance inflation factors (VIFs) of the predictors to ensure that they met the assumptions for the planned regression analyses. ${ }^{56}$ We examined the univariate associations between the demographic variables of age (using Pearson correlations) and sex (using one sample $t$-tests) and the study criterion variables (depression and physical function) to determine if either or both of these should be controlled in the planned regression analyses. Then, we computed Pearson correlation coefficients to assess the univariate associations between the socioeconomic (income and education) and psychological (pain catastrophizing and resilience) predictor variables and criterion variables.

Next, in order to test the study hypotheses, we performed two linear regression analyses, using the measures of physical function and depression as the criterion variables. In addition to entering any demographic variables (age or sex) that demonstrated significant univariate associations with the criterion variables, we entered pain intensity in the first step to control for the potential confounding effects of pain intensity on both the predictor and criterion variables. In the second step, we entered the socioeconomic predictor variables (education level and income). In the third step, we entered psychological predictor variables (pain catastrophizing and resilience). In the fourth and final step, we entered the six interaction terms representing the Pain Intensity $\times$ Catastrophizing and Pain intensity $\times$ Resilience Interaction effects, Income $\times$ Catastrophizing and Income $\times$ Resilience Interaction effects, and Education $\times$ Catastrophizing and Education $\times$ Resilience, stepwise. We mean centered the variables involved in the interactions (income, education, pain intensity, resilience, and catastrophizing) to limit multicollinearity before entering these variables in the regression model. We planned to interpret any significant interaction effects that emerged by examining the univariate associations between the predictor (pain catastrophizing or resilience) and criterion variables separately for those with high (above the median) vs low (below the median) scores on the moderating variable involved in the interaction. All analyses were performed using SPSS version 24, and we used an alpha level of 0.05 to identify an effect as statistically significant.

\section{Results}

\section{Description of the study participants}

A total of 143 participants were enrolled into the study; 100 $(69 \%)$ were recruited from the community and 43 (31\%) were recruited from the hospital. Table 1 presents the descriptive

Table I Description of the study participants

\begin{tabular}{|c|c|c|}
\hline Variables & Mean (SD) & n (\%) \\
\hline Age in years & $47.06(14.45)$ & \\
\hline Duration of pain in months & $51.10(76.15)$ & \\
\hline \multicolumn{3}{|l|}{ Study site } \\
\hline Rural community & & $100(69)$ \\
\hline Tertiary hospital & & $43(31)$ \\
\hline \multicolumn{3}{|l|}{ Sex } \\
\hline Men & & $50(35)$ \\
\hline Women & & $93(65)$ \\
\hline \multicolumn{3}{|l|}{ Religion } \\
\hline Hindu & & $132(92)$ \\
\hline Buddhist & & $5(4)$ \\
\hline Others & & $6(4)$ \\
\hline \multicolumn{3}{|l|}{ Race/ethnicity } \\
\hline Chettri & & $59(4 I)$ \\
\hline Brahmin & & $40(28)$ \\
\hline Newar & & $19(13)$ \\
\hline Tamang, Rai, Limbu & & $7(5)$ \\
\hline Dalit & & $7(5)$ \\
\hline Others & & $11(8)$ \\
\hline \multicolumn{3}{|l|}{ Education } \\
\hline No school & & $45(3 \mathrm{I})$ \\
\hline Primary school ( $<5$ years) & & $42(30)$ \\
\hline Secondary school (6-10 years) & & $35(24)$ \\
\hline Higher secondary school (I I-12 years) & & $5(4)$ \\
\hline Bachelor & & $13(9)$ \\
\hline Master and above & & $3(2)$ \\
\hline \multicolumn{3}{|l|}{ Occupation } \\
\hline Agriculture & & $49(34)$ \\
\hline Household work & & $39(27)$ \\
\hline Business & & $15(10)$ \\
\hline Office & & $11(8)$ \\
\hline Of working age but unemployed & & $7(5)$ \\
\hline Others & & $22(15)$ \\
\hline \multicolumn{3}{|l|}{$\begin{array}{l}\text { Monthly income (in Nepalese } \\
\text { rupees) }\end{array}$} \\
\hline No income & & $21(15)$ \\
\hline$\leq 10,000$ & & $47(33)$ \\
\hline $10,001-30,000$ & & $46(32)$ \\
\hline $30,00 \mathrm{I}-50,000$ & & $16(11)$ \\
\hline $50,001-100,000$ & & $6(4)$ \\
\hline$>100,000$ & & $4(3)$ \\
\hline Missing & & $3(2)$ \\
\hline \multicolumn{3}{|l|}{ Site of pain } \\
\hline Multiple sites & & $60(42)$ \\
\hline Low back and pelvis & & $32(22)$ \\
\hline Knee & & $30(2 \mathrm{I})$ \\
\hline Shoulder & & $8(6)$ \\
\hline Neck and upper extremity & & $8(5)$ \\
\hline Others & & $5(4)$ \\
\hline
\end{tabular}


information about the study sample, and Table 2 presents the mean and standard deviation values of the study variables with results of associations of the study variables. The majority of the study sample were women (65\%) and Hindu (92\%) and had relatively few years of formal education $(61 \%$ of the sample had no more than 5 years of education). Not all the participants completed all the measures; the number of participants who completed the scales is reported in Table 2. We excluded missing items from all analyses.

\section{Assumption testing}

The evaluation of the distributions of the study variables indicated that they were all adequately normal for the planned regression analyses (skewness range $=-2.13-0.91$, kurtosis range $=-0.64-0.30)$. The VIF statistics were all well below the cutoff value of 10 for VIF (range, 1.27-1.36), indicating that multicollinearity would not bias the findings. ${ }^{56}$

\section{Univariate correlations of control and predictor variables with criterion variables}

The univariate correlations among the study variables are presented in Table 2. As can be seen, neither age nor sex was significantly associated with any of the criterion variables. Therefore, they were not entered as control variables in the regression analyses. Income evidenced a weak (but statistically significant) association with depression, whereas resilience and catastrophizing showed a moderate and significant association with depression (Table 2). Education correlated significantly with physical function but not depression.

\section{Regression analysis}

\section{Predicting physical function}

The results of the regression analyses predicting physical function are presented in Table 3. Pain intensity did not make a statistically significant contribution to the prediction of physical function in the first step. As can be seen, both education $(\beta=0.31, P=0.001)$ and income $(\beta=-0.19, P=0.030)$ made significant contributions to the prediction of physical function. However, neither pain catastrophizing nor resilience made statistically significant independent contributions to the prediction of physical function, once education level, income, and pain intensity were controlled. Finally, both pain intensity $(\beta=0.20, P=0.029)$ and income $(\beta=0.20, P=0.023)$ moderated the association between resilience and physical function.

To interpret the significant interaction effects, we computed the zero-order correlations between resilience and physical function for participants reporting 1) more (above the sample's pain intensity median, ie, $\geq 6$ on the $0-10$ NRS; $\mathrm{n}=52, r=0.22, P=0.126$ ) vs less (below the sample's pain intensity median, ie, $<6$ on the $0-10$ NRS; $n=83, r=-0.02$, $P=0.870$ ) and 2 ) higher (ie, $\geq 10,000$ rupees in annual income; $\mathrm{n}=69 ; r=0.39, P=0.002$ ) vs lower (ie, $<10,000$ rupees in annual income; $\mathrm{n}=79, r=0.11, P=0.373$ ) income.

\section{Predicting depression}

With respect to the prediction of depression, only income emerged as statistically significant $(\beta=-0.20, P=0.036)$ in predicting depression in the first step. Pain catastrophizing ( $\beta=0.53, P<0.001)$, but not resilience $(\beta=-0.13, P=0.112)$, made a statistically significant independent contribution to the prediction of depression once income, pain intensity, and education were controlled. In addition, a statistically significant moderating effect of income on the associations between 1) resilience and depression (Income $\times$ Resilience interaction $\beta=-0.17, P=0.021$ ) and 2) catastrophizing and depression (Income $\times$ Catastrophizing interaction $\beta=-0.19$, $P=0.017$ ) emerged. The univariate correlations computed to interpret the significant interaction effect indicated a stronger negative association between resilience and depression

Table 2 Mean and SD values of the study variables and correlation coefficients between study variables

\begin{tabular}{|c|c|c|c|c|c|c|}
\hline Variables & $\mathbf{n}$ & Mean (SD) & NRS for pain & CD-RISC & BDI & PSFS \\
\hline Catastrophizing (PCS) & 143 & $23.19(11.68)$ & $0.2 \mathrm{I}^{\mathrm{a}}$ & $-0.46^{b}$ & $0.59^{\mathrm{b}}$ & -0.05 \\
\hline Average pain intensity (NRS) & 140 & $5.48(1.37)$ & & 0.01 & 0.11 & -0.06 \\
\hline Resilience (CD-RISC) & 140 & $5.43(1.94)$ & & & $-0.3 I^{b}$ & 0.08 \\
\hline Depression (BDI) & $14 \mid$ & $13.27(10.00)$ & & & & $0.29^{b}$ \\
\hline Physical function (PSFS) & 129 & $15.72(6.99)$ & & & & \\
\hline Income & 143 & & & & $-0.20^{\mathrm{a}}$ & -0.08 \\
\hline Education & 143 & & & & -0.02 & $0.26^{\circ}$ \\
\hline Age & $|4|$ & & & & 0.01 & -0.10 \\
\hline Sex & $14 \mid$ & & & & 0.08 & -0.09 \\
\hline
\end{tabular}

Note: ${ }^{\mathrm{a} P}<0.05,{ }^{\mathrm{b}} \mathrm{P}<0.01$.

Abbreviations: NRS, Numerical Rating Scale; CD-RISC, Connor Davidson Resilience Scale; BDI, Beck Depression Inventory; PSFS, Patient-Specific Functional Scale; PCS, Pain Catastrophizing Scale. 
Table 3 Results of the linear regression analyses predicting physical function and depression from catastrophizing and resilience scores

\begin{tabular}{|c|c|c|c|c|c|}
\hline Steps and variables & Total $R^{2}$ & $\boldsymbol{R}^{2} \Delta$ & $F-R^{2} \Delta$ & $B$ to enter & $P$ \\
\hline \multicolumn{6}{|l|}{ Criterion variable: physical function } \\
\hline Step I: Control variable & 0.004 & 0.004 & 0.458 & & \\
\hline Average pain intensity $(C)$ & & & & -0.06 & 0.500 \\
\hline Step 2: Socioeconomic variables & 0.089 & 0.085 & 5.581 & & \\
\hline Education (C) & & & & 0.31 & 0.001 \\
\hline Income (C) & & & & -0.19 & 0.030 \\
\hline Step 3: Psychological predictors & 0.091 & 0.002 & 0.129 & & \\
\hline Catastrophizing (C) & & & & -0.05 & 0.614 \\
\hline Resilience (C) & & & & -0.02 & 0.818 \\
\hline \multicolumn{6}{|l|}{ Step 4: Interaction terms } \\
\hline Pain Intensity $\times$ Resilience & 0.128 & 0.037 & 4.889 & 0.20 & 0.029 \\
\hline Income $\times$ Resilience & 0.166 & 0.038 & 5.274 & 0.20 & 0.023 \\
\hline \multicolumn{6}{|l|}{ Criterion variable: depression } \\
\hline Step I: Control variable & 0.013 & 0.013 & 1.733 & & \\
\hline Average pain intensity $(C)$ & & & & 0.11 & 0.190 \\
\hline Step 2: Socioeconomic variables & 0.046 & 0.033 & 2.236 & & \\
\hline Education (C) & & & & 0.07 & 0.439 \\
\hline Income $(\mathrm{C})$ & & & & -0.20 & 0.036 \\
\hline Step 3: Psychological predictors & 0.351 & 0.305 & 30.138 & & \\
\hline Catastrophizing (C) & & & & 0.53 & $<0.001$ \\
\hline Resilience (C) & & & & -0.13 & 0.112 \\
\hline \multicolumn{6}{|l|}{ Step 4: Interaction terms } \\
\hline Income $\times$ Resilience & 0.348 & 0.027 & 5.433 & -0.17 & 0.021 \\
\hline Income $\times$ Catastrophizing & 0.405 & 0.027 & 5.824 & -0.19 & 0.017 \\
\hline
\end{tabular}

Note: $(C)$, centered variables.

for participants reporting lower income (ie, $\mathrm{n}=69, r=-0.38$, $P=0.002)$, relative to those reporting higher income $(\mathrm{n}=71$, $r=-0.23, P=0.053)$.

\section{Discussion}

The key finding from this study was that pain catastrophizing, but not resilience, evidenced a direct strong association with depression, whereas neither factor evidenced a direct association with physical function in a sample of individuals with chronic pain from rural Nepal. In addition, monthly family income moderated the relationships between both catastrophizing and depression and resilience and depression; pain intensity and income moderated the relationships between resilience and physical function. These findings have important theoretical and clinical implications with respect to the generalizability of research findings derived from samples of individuals with chronic pain from developed countries, as well as the potential utility and efficacy of chronic pain treatments used in developed countries for treating pain in individuals from developing countries, such as Nepal.

\section{Predicting physical function}

Inconsistent with the study hypotheses, neither pain catastrophizing nor resilience predicted physical function in our sample; however, both education and income were weakly to moderately (but significantly) associated with this criterion variable. The findings related to pain catastrophizing are consistent with the prospective studies from Spain and the UK, which found that pain catastrophizing did not predict disability in individuals with chronic pain from those countries. ${ }^{10-12,57}$ On the other hand, a number of systematic reviews have concluded that pain catastrophizing is more likely to be associated with physical function samples from many Western countries. ${ }^{10-12}$ For example, majority of research on a sample of individuals with chronic pain shows that those who catastrophize about pain tend to be less active and endorse more physical disability across a variety of pain problems. ${ }^{30,58-64}$ Specifically, pain catastrophizing has been shown to be associated with disability in samples of individuals with mixed chronic pain, ${ }^{30,61,65}$ chronic anterior knee pain, ${ }^{58}$ and chronic low back pain ${ }^{66-68}$ from many developed countries.

Our null findings with respect to the prediction of physical function in the current study are intriguing, given their lack of consistency with many - but not all - previous research findings. One possible explanation could be due to the fact that the majority of the participants in this study live in rural and semi-urban communities of Nepal and have extremely low SES; many are farmers, and none have the "safety" net 
available to individuals in more developed countries. Thus, if they become so disabled that they are unable to work for themselves (eg, as farmers) or work for someone else for income, they would be unable to obtain the resources needed for basic food and shelter. Similarly, and relatedly, very few individuals in Nepal - including and especially those with low SES - have basic health benefits (ie, medical insurance or paid sick leave policies) that allow them to take time off for pain-related problems. When people need to continue to function physically in order to survive, their level of catastrophizing or resilience may not have the opportunity to impact physical function.

A second possibility for our null findings with respect to the prediction of physical function is that, although we used a measure of physical function with demonstrated validity and reliability in individuals with musculoskeletal pain in Nepal, ${ }^{52}$ the specific measure used in this study has not previously been used as a criterion measure in studies examining catastrophizing or resilience. Thus, it is possible that catastrophizing or resilience may not be related to the most important physical activities identified by the study participants that they have trouble performing due to pain and may instead be related to the physical function domains assessed by other measures. However, the measure of physical function used here did evidence significant positive associations with education and negative associations with income in our sample (eg, those with more relative income and higher education levels reported higher levels of physical function). These positive findings support the validity of the measure of physical function used here, and the finding with respect to education is consistent with analyses using participants from Korea with lumbar spinal stenosis, ${ }^{69}$ African-Americans and Caucasians with chronic pain from the USA, ${ }^{70}$ women with chronic pelvic pain from the USA, ${ }^{70}$ and a community sample of individuals with chronic pain from the USA. ${ }^{71}$ Thus, whether or not the null findings with respect to the associations between catastrophizing, resilience, and physical function replicates when other measures of physical function are used in Nepalese samples would need to be determined by additional research.

The positive finding with respect to education level as a predictor of physical function in this study is consistent with research studying a variety of health-related outcomes in samples from different countries and cultures. For example, lower levels of education has been shown to be associated with more episodes of back pain as well as poorer outcomes of both surgical and nonsurgical treatments. ${ }^{72,73}$ Likewise, having more education is associated with the use of adaptive pain-coping strategies, ${ }^{74}$ perhaps because individuals with more education may have more health literacy, including the understanding that being more active despite pain is adaptive and useful. ${ }^{74}$ If future research replicates the current findings with respect to the association between education level and function in Nepalese individuals, and health literacy is found to mediate this association, this would point to a specific intervention (ie, pain education ${ }^{75}$ ), which could potentially improve health literacy as a way to improve function in this population.

Another interesting finding is the weak negative (but still statistically significant) association between income level and physical function; those reporting lower family income also tended to endorse higher levels of physical function. This finding is consistent with the idea, introduced previously, that those with lower income levels may need to keep themselves physically active (either in the fields or at other work) in order to obtain the money needed to have the basic resources of food and shelter; those with more income may have more flexibility for being able to be less active when or if they develop a pain problem.

We also found that pain intensity did not correlate significantly with physical function in the current sample. This is inconsistent with research using participants from other countries $^{76}$ and another sample from Nepal ${ }^{52}$ but is consistent with a previous study that found that individuals with a low back pain from Nepal reporting more intense pain did not have more physical disability. ${ }^{77}$ Again, this finding may be due, at least in part, to the fact that the individuals in our sample with a very low education and income may be unable to be disabled and still obtain what they need to survive, regardless of their pain intensity. Overall, the findings indicate that the factors that contribute to physical function in individuals with a very low SES and education level may be different than those that contribute to physical function in individuals with more resources. Such individuals could potentially benefit more from treatments or interventions that address their basic needs (eg, education, job training) than from treatments that target catastrophizing or resilience; research examining this possibility is warranted.

\section{Predicting depression}

As hypothesized, pain catastrophizing is a strong predictor of depressive symptoms. A very large body of research supports a strong association between pain catastrophizing and depression across a wide variety of pain conditions and in samples of individuals from many different countries, although all the previous research in this area has been 
conducted in developed countries. ${ }^{30,43,78,79}$ This finding has important implications. First, this finding in a sample of individuals from a low-income country provides additional support for the generalizability of this association across cultures. It also suggests the possibility that treatments used in developed countries that have been shown to reduce pain catastrophizing (eg, cognitive behavioral therapy, ${ }^{80}$ pain education, ${ }^{75}$ and exposure therapy ${ }^{81}$ ) should be evaluated to determine if they might be effective for reducing catastrophizing, and subsequently improving depression, in developing countries.

The significant moderating effect of income on the associations between both 1) resilience and depression and 2) catastrophizing and depression in the current sample is important to consider. Resilience evidenced a stronger (and significant) association with depression in those with a higher income and a weaker association in those with a lower income, although resilience evidenced a negative association with depression in participants from both income groups. Thus, resilience appears to play a role in (or at least be a predictor of) depression in the current sample of individuals from Nepal, but it appears to be less important than catastrophizing. Perhaps for factors such as resilience that show marginal (small to medium) associations with function, their effects might only begin to emerge in individuals who might have more financial resources. Thus, it might be anticipated that interventions that target resilience as a way to improve adjustment to pain might be more effective for individuals in Nepal with more income. Research to examine this possibility is warranted.

\section{Limitations}

The findings of the current study have a number of limitations that should be considered when interpreting the results. First, it used cross-sectional data, which limits the conclusions that can be drawn with respect to causal relationships. However, the lack of significant associations between catastrophizing and resilience and physical function found here suggests the possibility that neither of these factors plays a causal role in physical function, because a significant association is a necessary (but not sufficient) condition for causal associations. Thus, if these psychosocial factors are important to function in Nepalese, the current findings suggest that they would be important to psychological function only; future research should examine these relationships using longitudinal data to evaluate the causal influence of these factors on depression. A second limitation is that the resilience measure used here had marginal internal consistency in our sample, although it has evidenced adequate test-retest stability. ${ }^{34}$ Nevertheless, it is possible that a relatively low internal consistency may have attenuated some of the associations found in analyses that used this measure. Although the moderating effect of income level on the association between resilience and depression that emerged indicates that the measure of resilience used did contain valid variance, additional and potentially more reliable measures of resilience (eg, the 10-item CD-RISC) should be considered in future research. Finally, the etiology of pain such as osteoporosis ${ }^{82}$ or rheumatoid arthritis ${ }^{83}$ may have had an influence on the role that psychosocial factors have in the adjustment to chronic pain, which we did not consider in the current paper. Future research examining the cause of pain as a potential factor that influences the role of psychological variables on patient adjustment would be useful.

\section{Summary and conclusion}

We found that pain catastrophizing predicted depression but not physical function in a sample of individuals with chronic pain living in rural areas of Nepal, a low-income country in Southeast Asia. Resilience was also associated (negatively) with depression in the study sample, but this association was only statistically significant in participants with a relatively higher income. These results indicate that research examining the effects of treatments targeting both catastrophizing and resilience in individuals with chronic pain and depression from Nepal is warranted. However, if the null findings with respect to the roles that both catastrophizing and resilience on physical function replicate in other studies of Nepalese individuals, the findings indicate that targeting these factors may have little beneficial effects on physical function in individuals with chronic pain from Nepal. Thus, it may be important to provide treatments that target other modifiable factors (eg, education, income, exercise) when the goal is to improve physical function in this population.

\section{Acknowledgment}

The authors would like to acknowledge the International Association for the Study of Pain (IASP) for providing a Developing Countries Collaborative Research Grant to MPJ and SS, which supported the current study.

\section{Disclosure}

The authors report no conflicts of interest in this work.

\section{References}

1. Henderson M, Kidd BL, Pearson RM, White PD. Chronic upper limb pain: an exploration of the biopsychosocial model. $J$ Rheumatol. 2005;32(1):118-122. 
2. Truchon M. Determinants of chronic disability related to low back pain: towards an integrative biopsychosocial model. Disabil Rehabil. 2001;23(17):758-767.

3. Jull G, Sterling M. Bring back the biopsychosocial model for neck pain disorders. Man Ther. 2009;14(2):117-118.

4. Andrasik F, Flor H, Turk DC. An expanded view of psychological aspects in head pain: the biopsychosocial model. Neurol Sci. 2005;26(Suppl 2):s87-s91.

5. Covic T, Adamson B, Spencer D, Howe G. A biopsychosocial model of pain and depression in rheumatoid arthritis: a 12-month longitudinal study. Rheumatology (Oxford). 2003;42(11):1287-1294.

6. Sullivan MJL, Bishop SR, Pivik J. The Pain Catastrophizing Scale: Development and validation. Psychol Assess. 1995;7(4):524-532.

7. Burns LC, Ritvo SE, Ferguson MK, Clarke H, Seltzer Z, Katz J. Pain catastrophizing as a risk factor for chronic pain after total knee arthroplasty: a systematic review. J Pain Res. 2015;8:21-32.

8. Wertli MM, Burgstaller JM, Weiser S, Steurer J, Kofmehl R, Held U. Influence of catastrophizing on treatment outcome in patients with nonspecific low back pain: a systematic review. Spine (Phila Pa 1976). 2014;39(3):263-273.

9. Theunissen M, Peters ML, Bruce J, Gramke HF, Marcus MA. Preoperative anxiety and catastrophizing: a systematic review and metaanalysis of the association with chronic postsurgical pain. Clin J Pain. 2012;28(9):819-841.

10. Foster NE, Thomas E, Bishop A, Dunn KM, Main CJ. Distinctiveness of psychological obstacles to recovery in low back pain patients in primary care. Pain. 2010;148(3):398-406.

11. Moix J, Kovacs FM, Martín A, Plana MN, Royuela A; Spanish Back Pain Research Network. Catastrophizing, state anxiety, anger, and depressive symptoms do not correlate with disability when variations of trait anxiety are taken into account. a study of chronic low back pain patients treated in Spanish pain units [NCT00360802]. Pain Med. 2011;12(7):1008-1017.

12. Kovacs FM, Seco J, Royuela A, Corcoll-Reixach J, Peña-Arrebola A; Spanish Back Pain Research Network. The prognostic value of catastrophizing for predicting the clinical evolution of low back pain patients: a study in routine clinical practice within the Spanish National Health Service. Spine J. 2012;12(7):545-555.

13. Sturgeon JA, Zautra AJ. Resilience: a new paradigm for adaptation to chronic pain. Curr Pain Headache Rep. 2010;14(2):105-112.

14. Newton-John TR, Mason C, Hunter M. The role of resilience in adjustment and coping with chronic pain. Rehabil Psychol. 2014;59(3):360-365.

15. Sturgeon JA, Zautra AJ, Arewasikporn A. A multilevel structural equation modeling analysis of vulnerabilities and resilience resources influencing affective adaptation to chronic pain. Pain. 2014;155(2):292-298.

16. Ong AD, Zautra AJ, Reid MC. Psychological resilience predicts decreases in pain catastrophizing through positive emotions. Psychol Aging. 2010;25(3):516-523.

17. Bauer H, Emeny RT, Baumert J, Ladwig KH. Resilience moderates the association between chronic pain and depressive symptoms in the elderly. Eur J Pain. 2016;20(8):1253-1265.

18. Karoly P, Ruehlman LS. Psychological "resilience" and its correlates in chronic pain: findings from a national community sample. Pain. 2006;123(1-2):90-97.

19. Day MA, Thorn BE. The relationship of demographic and psychosocial variables to pain-related outcomes in a rural chronic pain population. Pain. 2010;151(2):467-474.

20. Cohen J, Cohen P, West SG, Aiken LS. Applied Multiple Regression/ Correlation Analysis for the Behavioral Sciences: New York: Routledge; 2013.

21. Edwards RR, Giles J, Bingham CO 3rd, Campbell C, Haythornthwaite JA, Bathon J. Moderators of the negative effects of catastrophizing in arthritis. Pain Med. 2010;11(4):591-599.

22. Suso-Ribera C, García-Palacios A, Botella C, Ribera-Canudas MV. Pain Catastrophizing and Its Relationship with Health Outcomes: Does Pain Intensity Matter? Pain Res Manag. 2017;2017:9762864.
23. Ferreira-Valente MA, Ribeiro JL, Jensen MP, Almeida R. Coping with chronic musculoskeletal pain in Portugal and in the United States: a cross-cultural study. Pain Med. 2011;12(10):1470-1480.

24. Ferreira-Valente MA, Pais-Ribeiro JL, Jensen MP. Associations between psychosocial factors and pain intensity, physical functioning, and psychological functioning in patients with chronic pain: a cross-cultural comparison. Clin J Pain. 2014;30(8):713-723.

25. Tan G, Jensen MP, Thornby J, Anderson KO. Ethnicity, control appraisal, coping, and adjustment to chronic pain among black and white Americans. Pain Med. 2005;6(1):18-28.

26. Jeong HS, Kang I, Namgung E, et al. Validation of the Korean version of the Connor-Davidson Resilience Scale-2 in firefighters and rescue workers. Compr Psychiatry. 2015;59:123-128.

27. Ni MY, Li TK, Yu NX, et al. Normative data and psychometric properties of the Connor-Davidson Resilience Scale (CD-RISC) and the abbreviated version (CD-RISC2) among the general population in Hong Kong. Qual Life Res. 2016;25(1):111-116.

28. Baek HS, Lee KU, Joo EJ, Lee MY, Choi KS. Reliability and validity of the Korean version of the Connor-Davidson resilience scale. Psychiatry Investig. 2010;7(2):109-115.

29. Ye ZJ, Qiu HZ, Li PF, et al. Validation and application of the Chinese version of the 10-item Connor-Davidson Resilience Scale (CD-RISC-10) among parents of children with cancer diagnosis. Eur J Oncol Nurs. 2017;27:36-44.

30. Yap JC, Lau J, Chen PP, et al. Validation of the Chinese Pain Catastrophizing Scale (HK-PCS) in patients with chronic pain. Pain Med. 2008;9(2):186-195.

31. Higuchi D. Prognostic Value of Preoperative Coping Strategies for Pain in Patients with Residual Neuropathic Pain after Laminoplasty for Compressive Cervical Myelopathy. Asian Spine J. 2015;9(5): 675-682.

32. Sharma P, Guha-Khasnobis B, Raj Khanal D. Nepal Human Development Report 2014. Kathmandu: GoN (Government of Nepal) and UNDP (United Nations Development Programme); 2014.

33. Government of Nepal. National Population and Housing Census 2011 (National Report). Central Bureau of Statistics, editor. Vol. 1. Kathmandu: NHPC; 2012.

34. Sharma S, Pathak A, Abbott JH, Jensen MP. Measurement properties of the Nepali version of the Connor Davidson resilience scales in individuals with chronic pain. Health Qual Life Outcomes. 2018;16(1):56.

35. Sharma S, Thibault P, Abbott JH, Jensen MP. Clinimetric properties of the Nepali version of the Pain Catastrophizing Scale in individuals with chronic pain. J Pain Res. 2018;11:265-276.

36. Margolis RB, Chibnall JT, Tait RC. Test-retest reliability of the pain drawing instrument. Pain. 1988;33(1):49-51.

37. Dworkin RH, Turk DC, Farrar JT, et al. Core outcome measures for chronic pain clinical trials: IMMPACT recommendations. Pain. 2005;113(1-2):9-19.

38. Werneke M, Hart DL, Cook D. A Descriptive Study of the Centralization Phenomenon. Spine (Phila Pa 1976). 1999;24(7):676-683.

39. Barbero M, Moresi F, Leoni D, Gatti R, Egloff M, Falla D. Test-retest reliability of pain extent and pain location using a novel method for pain drawing analysis. Eur J Pain. 2015;19(8):1129-1138.

40. Sharma S, Palanchoke J, Reed D, Haxby Abbott J. Translation, crosscultural adaptation and psychometric properties of the Nepali versions of numerical pain rating scale and global rating of change. Health Qual Life Outcomes. 2017;15(1):236.

41. Hjermstad MJ, Fayers PM, Haugen DF, et al. Studies comparing Numerical Rating Scales, Verbal Rating Scales, and Visual Analogue Scales for assessment of pain intensity in adults: a systematic literature review. $J$ Pain Symptom Manage. 2011;41(6):1073-1093.

42. Chiarotto A, Boers M, Deyo RA, et al. Core outcome measurement instruments for clinical trials in nonspecific low back pain. Pain. 2018;159(3):481-495.

43. Cho S, Kim HY, Lee JH. Validation of the Korean version of the Pain Catastrophizing Scale in patients with chronic non-cancer pain. Qual Life Res. 2013;22(7):1767-1772. 
44. Meyer K, Sprott H, Mannion AF. Cross-cultural adaptation, reliability, and validity of the German version of the Pain Catastrophizing Scale. J Psychosom Res. 2008;64(5):469-478.

45. Vaishnavi S, Connor K, Davidson JR. An abbreviated version of the Connor-Davidson Resilience Scale (CD-RISC), the CD-RISC2: psychometric properties and applications in psychopharmacological trials. Psychiatry Res. 2007;152(2-3):293-297.

46. Beck AT, Ward CH, Mendelson M, Mock J, Erbaugh J. An inventory for measuring depression. Arch Gen Psychiatry. 1961;4:561-571.

47. Kohrt BA, Kunz RD, Koirala NR, Sharma VD, Nepal MK. Validation of a Nepali version of the Beck Depression Inventory. Nepalese Journal of Psychiatry. 2002;2(4):123-130.

48. Kapci EG, Uslu R, Turkcapar H, Karaoglan A. Beck Depression Inventory II: evaluation of the psychometric properties and cut-off points in a Turkish adult population. Depress Anxiety. 2008;25(10):E104-E110.

49. Sacco R, Santangelo G, Stamenova S, et al. Psychometric properties and validity of Beck Depression Inventory II in multiple sclerosis. Eur J Neurol. 2016;23(4):744-750.

50. Abubakar A, Kalu RB, Katana K, et al. Adaptation and Latent Structure of the Swahili Version of Beck Depression Inventory-II in a Low Literacy Population in the Context of HIV. PLoS One. 2016;11(6):e0151030.

51. Kohrt BA, Speckman RA, Kunz RD, et al. Culture in psychiatric epidemiology: using ethnography and multiple mediator models to assess the relationship of caste with depression and anxiety in Nepal. Ann Hum Biol. 2009;36(3):261-280.

52. Sharma S, Palanchoke J, Abbott JH. Cross-cultural Adaptation and Validation of the Nepali Translation of the Patient-Specific Functional Scale. J Orthop Sports Phys Ther. 2018;48(8):1-23.

53. Stratford P, Gill C, Westaway M, Binkley J. Assessing Disability and Change on Individual Patients: A Report of a Patient Specific Measure. Physiotherapy Canada. 1995;47(4):258-263.

54. Horn KK, Jennings S, Richardson G, Vliet DV, Hefford C, Abbott JH. The patient-specific functional scale: psychometrics, clinimetrics, and application as a clinical outcome measure. J Orthop Sports Phys Ther. 2012;42(1):30-42.

55. Abbott JH, Schmitt JS. The Patient-Specific Functional Scale was valid for group-level change comparisons and between-group discrimination. J Clin Epidemiol. 2014;67(6):681-688.

56. Hair JF, Black WC, Babin BJ, Anderson RE, Tatham RL. Multivariate Data Analysis. Vol. 5. NJ, USA: Prentice Hall Upper Saddle River; 1998.

57. Albaladejo C, Kovacs FM, Royuela A, del Pino R, Zamora J; Spanish Back Pain Research Network. The efficacy of a short education program and a short physiotherapy program for treating low back pain in primary care: a cluster randomized trial. Spine (Phila Pa 1976). 2010;35(5):483-496.

58. Doménech J, Sanchis-Alfonso V, Espejo B. Changes in catastrophizing and kinesiophobia are predictive of changes in disability and pain after treatment in patients with anterior knee pain. Knee Surg Sports Traumatol Arthrosc. 2014;22(10):2295-2300.

59. Turner JA, Jensen MP, Warms CA, Cardenas DD. Catastrophizing is associated with pain intensity, psychological distress, and pain-related disability among individuals with chronic pain after spinal cord injury. Pain. 2002;98(1-2):127-134.

60. Vangronsveld KL, Peters M, Goossens M, Vlaeyen J. The influence of fear of movement and pain catastrophizing on daily pain and disability in individuals with acute whiplash injury: a daily diary study. Pain. 2008;139(2):449-457.

61. Arnow BA, Blasey CM, Constantino MJ, et al. Catastrophizing, depression and pain-related disability. Gen Hosp Psychiatry. 2011;33(2):150-156.

62. Kovacs FM, Seco J, Royuela A, Peña A, Muriel A, Spanish Back Pain Research Network. The correlation between pain, catastrophizing, and disability in subacute and chronic low back pain: a study in the routine clinical practice of the Spanish National Health Service. Spine (Phila Pa 1976). 2011;36(4):339-345.
63. Parr JJ, Borsa PA, Fillingim RB, et al. Pain-related fear and catastrophizing predict pain intensity and disability independently using an induced muscle injury model. J Pain. 2012;13(4):370-378.

64. Whyte A, Carroll LJ. The relationship between catastrophizing and disability in amputees experiencing phantom pain. Disabil Rehabil. 2004;26(11):649-654.

65. Severeijns R, Vlaeyen JW, van den Hout MA, Weber WE. Pain catastrophizing predicts pain intensity, disability, and psychological distress independent of the level of physical impairment. Clin J Pain. 2001;17(2):165-172.

66. Thomas EN, Pers YM, Mercier G, et al. The importance of fear, beliefs, catastrophizing and kinesiophobia in chronic low back pain rehabilitation. Ann Phys Rehabil Med. 2010;53(1):3-14.

67. Picavet HS, Vlaeyen JW, Schouten JS. Pain catastrophizing and kinesiophobia: predictors of chronic low back pain. Am J Epidemiol. 2002;156(11):1028-1034.

68. Bansal D, Gudala K, Lavudiya S, Ghai B, Arora P. Translation, Adaptation, and Validation of Hindi Version of the Pain Catastrophizing Scale in Patients with Chronic Low Back Pain for Use in India. Pain Med. 2016;17(10):1848-1858.

69. Kim HJ, Kim SC, Kang KT, Chang BS, Lee CK, Yeom JS. Influence of educational attainment on pain intensity and disability in patients with lumbar spinal stenosis: mediation effect of pain catastrophizing. Spine (Phila Pa 1976). 2014;39(10):E637-644.

70. Roth RS, Punch MR, Bachman JE. Educational achievement and pain disability among women with chronic pelvic pain. J Psychosom Res. 2001;51(4):563-569.

71. Cats-Baril WL, Frymoyer JW. Demographic factors associated with the prevalence of disability in the general population. Analysis of the NHANES I database. Spine (Phila Pa 1976). 1991;16(6):671-674.

72. Dionne CE, Von Korff M, Koepsell TD, Deyo RA, Barlow WE, Checkoway H. Formal education and back pain: a review. J Epidemiol Community Health. 2001;55(7):455-468.

73. Dionne C, Koepsell TD, Von Korff M, Deyo RA, Barlow WI, Checkoway H. Formal education and back-related disability. In search of an explanation. Spine (Phila Pa 1976). 1995;20(24):2721-2730.

74. Roth RS, Geisser ME. Educational achievement and chronic pain disability: mediating role of pain-related cognitions. Clin J Pain. 2002;18(5):286-296.

75. Moseley GL, Butler DS. Fifteen Years of Explaining Pain: The Past, Present, and Future. J Pain. 2015;16(9):807-813.

76. Costa LO, Maher CG, Latimer J, et al. Clinimetric testing of three selfreport outcome measures for low back pain patients in Brazil: which one is the best? Spine (Phila Pa 1976). 2008;33(22):2459-2463.

77. Anderson RT. An orthopedic ethnography in rural Nepal. MedAnthropol. 1984;8(1):46-59.

78. Fernandes L, Storheim K, Lochting I, Grotle M. Cross-cultural adaptation and validation of the Norwegian pain catastrophizing scale in patients with low back pain. BMC Musculoskelet Disord. 2012;13:111.

79. Monticone M, Baiardi P, Ferrari S, et al. Development of the Italian version of the Pain Catastrophising Scale (PCS-I): cross-cultural adaptation, factor analysis, reliability, validity and sensitivity to change. Qual Life Res. 2012;21(6):1045-1050.

80. Turk DC, Meichenbaum D, Genest M. Pain and Behavioral Medicine: A Cognitive-Behavioral Perspective. Vol. 1. New York: Guilford Press; 1983.

81. Vlaeyen JW, de Jong J, Geilen M, Heuts PH, van Breukelen G. The treatment of fear of movement/(re)injury in chronic low back pain: further evidence on the effectiveness of exposure in vivo. Clin J Pain. 2002;18(4):251-261.

82. Catalano A, Martino G, Morabito N, et al. Pain in Osteoporosis: From Pathophysiology to Therapeutic Approach. Drugs Aging. 2017;34(10):755-765.

83. Walsh DA, McWilliams DF. Mechanisms, impact and management of pain in rheumatoid arthritis. Nat Rev Rheumatol. 2014;10(10): $581-592$. 
The Journal of Pain Research is an international, peer reviewed, open access, online journal that welcomes laboratory and clinical findings in the fields of pain research and the prevention and management of pain. Original research, reviews, symposium reports, hypothesis formation and commentaries are all considered for publication.
The manuscript management system is completely online and includes a very quick and fair peer-review system, which is all easy to use. Visit http://www.dovepress.com/testimonials.php to read real quotes from published authors. 